\title{
Cost-Utility Analysis of Cochlear Implantation in Australian Adults
}

\author{
$* \dagger$ Chris Foteff, $*$ Steven Kennedy, $\dagger$ Abul Hasnat Milton, $\ddagger$ Melike Deger, \\ $*$ Florian Payk, and $*$ Georgina Sanderson
}

\begin{abstract}
*Cochlear Limited, Macquarie University, Sydney; †Centre for Clinical Epidemiology and Biostatistics (CCEB), School of Medicine and Public Health, The University of Newcastle, Callaghan, NSW, Australia; and $\ddagger$ Cochlear AG Europe, Europe Middle East and Africa Headquarters, Basel, Switzerland
\end{abstract}

Objectives: Sequential and simultaneous bilateral cochlear implants are emerging as appropriate treatment options for Australian adults with sensory deficits in both cochleae. Current funding of Australian public hospitals does not provide for simultaneous bilateral cochlear implantation (CI) as a separate surgical procedure. Previous cost-effectiveness studies of sequential and simultaneous bilateral CI assumed $100 \%$ of unilaterally treated patients' transition to a sequential bilateral CI. This assumption does not place cochlear implantation in the context of the generally treated population. When mutually exclusive treatment options exist, such as unilateral CI, sequential bilateral CI, and simultaneous bilateral CI, the mean costs of the treated populations are weighted in the calculation of incremental costutility ratios. The objective was to evaluate the cost-utility of bilateral hearing aids (HAs) compared with unilateral, sequential, and simultaneous bilateral CI in Australian adults with bilateral severe to profound sensorineural hearing loss.

Research Design: Cost-utility analysis of secondary sources input to a Markov model.

Setting: Australian health care perspective, lifetime horizon with costs and outcomes discounted 5\% annually.
Intervention: Bilateral HAs as treatment for bilateral severe to profound sensorineural hearing loss compared with unilateral, sequential, and simultaneous bilateral CI.

Main Outcome Measures: Incremental costs per quality adjusted life year (AUD/QALY).

Results: When compared with bilateral hearing aids the incremental cost-utility ratio for the CI treatment population was AUD11,160/QALY. The incremental cost-utility ratio was weighted according to the number of patients treated unilaterally, sequentially, and simultaneously, as these were mutually exclusive treatment options.

Conclusion: No peer-reviewed articles have reported the incremental analysis of cochlear implantation in a continuum of care for surgically treated populations with bilateral severe to profound sensorineural hearing loss. Unilateral, sequential, and simultaneous bilateral CI were cost-effective when compared with bilateral hearing aids. Technologies that reduce the total number of visits for a patient could introduce additional cost efficiencies into clinical practice.

Key Words: Adults—Australia-Bilateral cochlear implantCost effectiveness-Cost utility-Economic evaluationSequential-Simultaneous-Unilateral.

Otol Neurotol 37:454-461, 2016.
Hearing loss is a complex, chronic condition (1) caused by many factors and can affect an individual at any time (2). Natural hearing with two ears enables sound localization and better speech understanding in both quiet and noisy environments. Adults may be treated with unilateral, sequential, or simultaneous bilateral cochlear implantation (CI), with patient-specific rehabilitation for improved hearing, communication, and spatial awareness (3). Sequential and simultaneous bilateral CI stimulates the auditory neural pathways delivering an artificial binaural experience (2). Superior sound localization and speech discrimination in noise are experienced by
Address correspondence and reprint requests to Chris Foteff, M.Clin.Epid., Cochlear Limited, 1 University Avenue, Macquarie University, Sydney, NSW 2109, Australia; E-mail: cfoteff@icloud.com.

C.F., S.K., M.D., F.P., and G.S. are employees of Cochlear Limited. C.F. is currently employed at Medtronic Inc. A.H.M. is an academic at the Centre for Clinical Epidemiology and Biostatistics, School of Medicine and Public Health, The University of Newcastle, Callaghan, Australia and declares no competing interests.

S.K. and C.F. designed and performed the Markov model and analyzed data; S.K. performed the Monte Carlo simulations and C.F. analyzed the output; C.F. wrote the paper; F.P. collected and analyzed data from
Cochlear Limited; M.D. reviewed the model; M.D., A.H.M., and G.S. provided critical input into research design; all authors provided critical revision of the manuscript and approved the paper.

This research received no funding. It was undertaken as an employment objective by C.F. All authors maintained independent control of manuscript content and were not subject to any censorship or editorial control by Cochlear Limited.

This is an open access article distributed under the Creative Commons Attribution License 4.0, which permits unrestricted use, distribution, and reproduction in any medium, provided the original work is properly cited. 
adults with sequential or simultaneous bilateral implants when compared with unilateral implants and bilateral hearing aids (HAs) (4).

CI procedures are safe, effective, and cost-effective (5-7). Sequential and simultaneous bilateral CI may be reimbursed in Australia by private health insurance funds or the Australian Department of Veteran Affairs, according to the approved indications on the Australian Register of Therapeutic Goods (8). Capped funding in the Australian public hospital system limits the number of CI surgeries available for adults, which means most adults in Australia with bilateral severe to profound sensorineural hearing loss (SP SNHL) are treated with unilateral CI $(9,10)$.

Published evidence confirms the value of sequential and simultaneous bilateral $\mathrm{CI}$ in the treatment of bilateral SP SNHL for adults $(7,11)$. The National Institute for Care and Health Excellence (NICE) and the Washington State Healthcare Authority commissioned comprehensive evaluations of unilateral, sequential bilateral, and simultaneous bilateral CIs in adults $(12,13)$. NICE concluded in their guidance of 2009 that unilateral CI and sequential or simultaneous bilateral CI were cost effective in children. NICE did not support reimbursement of sequential or simultaneous bilateral CIs for adults without comorbidities (12). In 2013, the Washington State Medicaid Scheme approved coverage of sequential and simultaneous bilateral CI for children and adults (13). Qualifying adults and children are now able to access sequential or simultaneous bilateral CI through the Washington State Medicaid Scheme (14). The most recent economic evaluation of CI in adults compared sequential bilateral CI with no intervention in the Canadian context. Their study demonstrated the costeffectiveness of sequential bilateral $\mathrm{CI}$ in adults in defined settings (11).

There is a need to evaluate the cost-utility of unilateral, sequential bilateral, and simultaneous bilateral CI for adults with postlingual bilateral SP SNHL in the Australian context.

In this cost-utility analysis Markov model techniques informed the economic evaluation, incorporating costs and stated health utilities. A Markov model permits evaluation of multiple treatment alternatives as health states and simulates the progress of a patient as they transition from one health state to the next or stay within a specific health state (15). Utility is the term used to describe the stated health preference of an individual measured with different survey methods. Perfect health is considered a score of one whereas death is equal to zero. The advantage of a Markov model is that it places treatments in the context with alternative treatments. Previous economic evaluations assumed $100 \%$ of unilaterally treated patients would transition to a sequential bilateral CI $(5,11,16)$.

Australian data was used in this cost-utility analysis to identify the proportion of adults who transitioned from bilateral HAs to each CI health state (10). The economic question of interest was whether CICI as a surgical intervention is cost effective when compared with bilateral HAs including all available mutually exclusive and exhaustive configurations of CI.

The objective was to evaluate the cost-utility of bilateral HAs compared with unilateral, sequential bilateral, and simultaneous bilateral CI in Australian adults with bilateral SP SNHL.

\section{METHODS}

This publication is structured according to the Consolidated Health Economic Evaluation Reporting Standards (17).

\section{Target Population and Subgroups}

Adults with postlingual bilateral SP SNHL eligible for CI were the populations of interest. These included adults with clinical presentations for unilateral CI, such as asymmetric SP SNHL, adults whose clinical needs warranted sequential bilateral CI, such as progressive hearing loss in the nonimplanted ear, and adults with clinical presentations for unambiguous simultaneous bilateral CI, such as sudden bilateral SP SNHL.

This analysis compares adults treated with nonsurgical bilateral HAs with adults treated surgically with CI. Unilateral $\mathrm{CI}$, sequential bilateral $\mathrm{CI}$, and simultaneous bilateral CI were evaluated as part of the mutually exclusive and exhaustive CI surgical treatment options available in a continuum of care provided to adults with sensory organ deficits in two cochleae.

Adults with bimodal devices were not included in this evaluation because no published data existed at the time of publication to inform the proportion of Australian adults unilaterally implanted who also wore an HA in their contralateral ear. No stated health preferences were published for this adult group.

\section{Setting and Location}

Approximately 75\% of CI surgeries in Australia in 2013 were performed on adults and approximately $60 \%$ were performed in the private hospital setting $(10,18)$.

\section{Study Perspective}

The base case analysis was conducted from the Australian health care system. Tangible surgery costs, audiology costs, and rehabilitation costs were included in the analysis. No costs borne by the patient or immediate family were included, such as time off work, or travel to and from clinic visits.

\section{Comparators}

Adults treated nonsurgically with bilateral HAs and adults treated surgically with unilateral CIs, sequential bilateral CIs, or simultaneous bilateral CIs as depicted in Figure 1.

\section{Time Horizon}

Costs and consequences were evaluated in the base case over a lifetime with sound processor replacements occurring every 5 years. The model started at age 18 and simulated CI surgeries according to age and transition probabilities. Australian life tables informed the all-cause mortality rate for the health states. One cycle in the Markov model was 1 year.

\section{Discount Rate}

Costs and outcomes were discounted at $5 \%$ annually which is the standard rate for Australian economic evaluations $(15,18)$. 


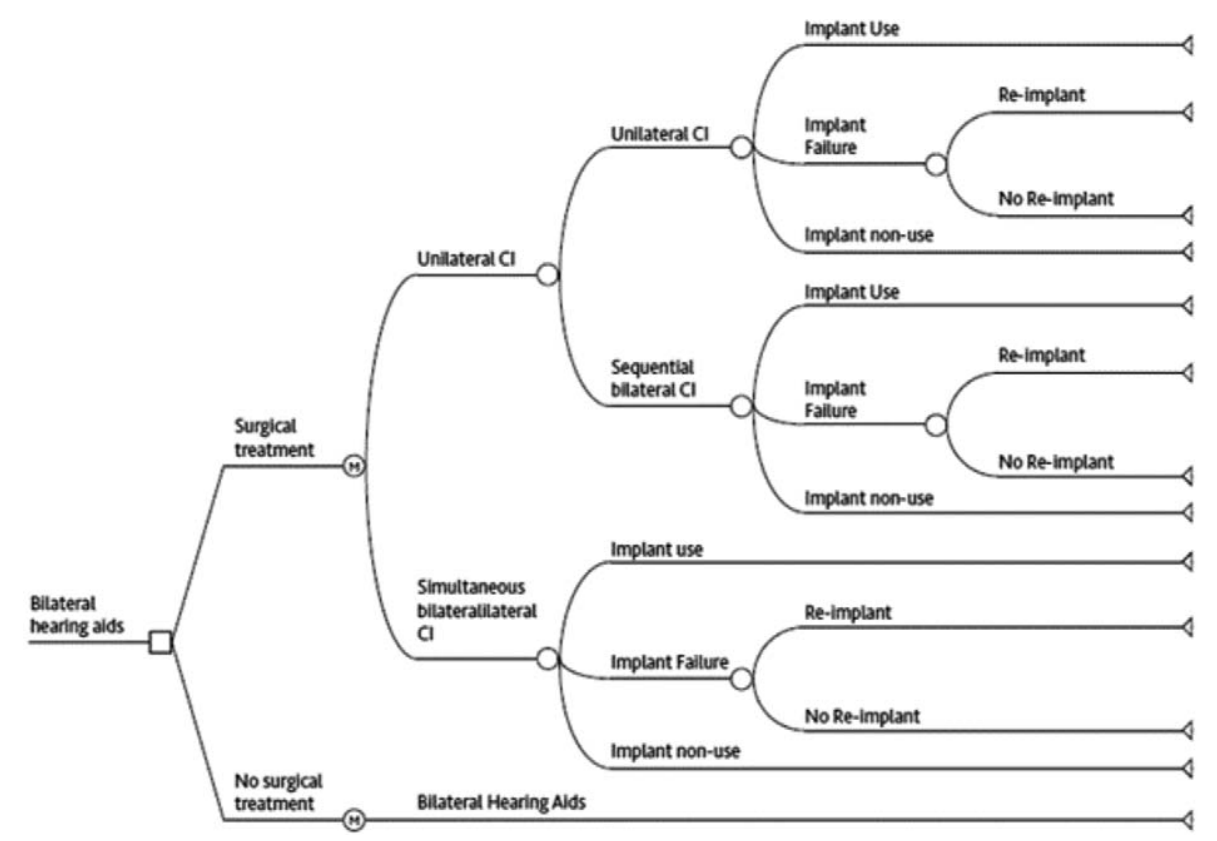

FIG. 1. Markov model structure.

\section{Choice of Health Outcomes}

The incremental cost per quality adjusted life year (AUD/ QALY) gained during a patient's lifetime.

\section{MEASUREMENTS OF EFFECT-RESOURCES AND COST ESTIMATES}

In the base case costs were based on published Medicare Benefits Schedule item numbers for relevant procedure codes as on July 1, 2013 (18). Prostheses costs were based on the Australian Prostheses List Billing Codes published as on August 28, 2013 for a cochlear implant and sound processor and the National Hospital Cost Data Collection Australian Public Hospitals Cost Report 2011 to 2012, Round 16, Appendix B (actual) for AR-DRG version 6.0x informed hospital costs (19).

The model considered presurgery assessment costs including specialist consultations, audiological hearing assessments, audiological speech assessments, and vestibular tests. Surgical costs included direct and overhead costs for ward, nursing, other clinical staff, pharmacy, imaging, theater, hospital bed costs, as well as implant and sound processor costs. Postsurgery costs included fitting and programming of the sound processor, specialist follow-up consultations, audiological hearing assessments, speech assessments, ongoing maintenance over time including outpatient costs, spares and repairs, failure rates, nonuse of the implant, and sound processor replacement costs every 5 years $(19-21)$.

On the basis of expert opinion, it was assumed adults would attend eight preassessment visits, including with surgeons and audiologists for speech and hearing assessments, and 12 postsurgical visits in the first year including two visits with surgeons for follow-up care, and 10 visits with audiologists for specific hearing and speech therapy. It was assumed all patients would attend one visit per year thereafter (expert opinion, personal communication, 2014).

Sequential bilateral implantation involves the cost of two separate hospital episodes and follow-up, whereas the surgical procedure costs of simultaneous bilateral CI occur during the same hospital admission. In Australia, a multiple surgery rule applies whereby 1.5 times the cost of the highest single procedure is covered for multiple procedures. This assumes cost efficiencies are introduced when multiple procedures are performed concurrently, such as when simultaneous bilateral CI is performed (18).

For the HA group, the cost of AUD 3,000 for an HA was the median retail price of a range of devices offered by the Australian Government Hearing Services Program (22). On the basis of expert opinion, it was assumed adults would attend two preassessment surgical visits to rule out surgical treatments, and two preassessment audiological visits; and then three audiological visits for fitting and follow-up in the first year (expert opinion, personal communication, 2014). It was assumed all patients would attend one visit per year thereafter.

\section{Explant and Replacement Rates in the Model}

Explant and reimplantation rates were identified from published data $(20,21)$. A cumulative annual failure rate of $1 \%$ was calculated on the basis of 30 years of CI experience at a major Australian CI clinic (21). Raine (20) reported 2 of 185 adults or $1.1 \%$ did not use their implant.

\section{Currency, Price, Date, and Conversion}

Costs were based on published fees as on July12, 2013 and are presented in Table 1. Currency was AUD. Unit costs are summarized in Table 1. 
TABLE 1. Base case costs

\begin{tabular}{|c|c|c|c|c|c|}
\hline & Unit Costs & Hearing Aids & Unilateral CI & $\begin{array}{l}\text { Sequential } \\
\text { Bilateral CI }\end{array}$ & $\begin{array}{l}\text { Simultaneous } \\
\text { Bilateral CI }\end{array}$ \\
\hline \multicolumn{6}{|l|}{ 1. Assessment costs } \\
\hline Specialist consultation-initial & $\$ 85.55$ & $\$ 85.55$ & $\$ 85.55$ & $\$ 171.10$ & $\$ 85.55$ \\
\hline Specialist consultation-subsequent & $\$ 43.00$ & $\$ 43.00$ & $\$ 94.60$ & $\$ 147.99$ & $\$ 55.18$ \\
\hline Audiology hearing assessment & $\$ 115.35$ & $\$ 230.70$ & $\$ 426.80$ & $\$ 667.00$ & $\$ 233.48$ \\
\hline Audiology speech assessment & $\$ 49.20$ & $\$ 0.00$ & $\$ 0.00$ & $\$ 0.00$ & $\$ 0.00$ \\
\hline Vestibular tests & $\$ 89.20$ & $\$ 0.00$ & $\$ 89.20$ & $\$ 89.20$ & $\$ 89.20$ \\
\hline Subtotal & & $\$ 359.25$ & $\$ 696.15$ & $\$ 1,075.29$ & $\$ 463.41$ \\
\hline \multicolumn{6}{|l|}{ 2. Implantation of the internal components } \\
\hline Direct costs-excl. prostheses & $\$ 6,398.65$ & $\$ 0.00$ & $\$ 6,398.65$ & $\$ 12,797.30$ & $\$ 9,597.97$ \\
\hline Overheads & $\$ 3,222.37$ & $\$ 0.00$ & $\$ 3,222.37$ & $\$ 6,444.73$ & $\$ 4,833.55$ \\
\hline Cochlear implant or cochlear hybrid implant & $\$ 13,500.00$ & $\$ 0.00$ & $\$ 13,500.00$ & $\$ 27,000.00$ & $\$ 27,000.00$ \\
\hline Cochlear sound processor-initial & $\$ 11,500.00$ & $\$ 0.00$ & $\$ 11,500.00$ & $\$ 23,000.00$ & $\$ 23,000.00$ \\
\hline Hearing aid & $\$ 3,000.00$ & $\$ 3,000.00$ & $\$ 0.00$ & $\$ 0.00$ & $\$ 0.00$ \\
\hline Subtotal & & $\$ 3,000.00$ & $\$ 34,621.01$ & $\$ 69,242.03$ & $\$ 64,431.52$ \\
\hline \multicolumn{6}{|l|}{ 3. Postoperative services } \\
\hline Follow-up consultation & $\$ 43.00$ & $\$ 43.00$ & $\$ 176.30$ & $\$ 192.90$ & $\$ 151.22$ \\
\hline Fitting of sound processor $(1 \mathrm{~h})$ & $\$ 192.45$ & $\$ 192.45$ & $\$ 192.45$ & $\$ 384.90$ & $\$ 288.68$ \\
\hline First 12 months' audiology assessments & $\$ 115.35$ & $\$ 230.70$ & $\$ 945.87$ & $\$ 1,813.86$ & $\$ 732.13$ \\
\hline First 12 months' speech assessments & $\$ 49.20$ & $\$ 0.00$ & $\$ 0.00$ & $\$ 0.00$ & $\$ 0.00$ \\
\hline Annual audiology follow-up for 5 years & $\$ 115.35$ & $\$ 461.40$ & $\$ 461.40$ & $\$ 922.80$ & $\$ 461.40$ \\
\hline Annual speech follow-up for 5 years & $\$ 49.20$ & $\$ 0.00$ & $\$ 0.00$ & $\$ 0.00$ & $\$ 0.00$ \\
\hline Replacement sound processor after 5 years & $\$ 8,050.00$ & $\$ 0.00$ & $\$ 8,050.00$ & $\$ 16,100.00$ & $\$ 16,100.00$ \\
\hline Replacement hearing aid after 5 years & $\$ 3,000.00$ & $\$ 3,000.00$ & $\$ 0.00$ & $\$ 0.00$ & $\$ 0.00$ \\
\hline Australian Hearing support for cables and batteries & $\$ 498.00$ & $\$ 0.00$ & $\$ 0.00$ & $\$ 0.00$ & $\$ 0.00$ \\
\hline Subtotal & & $\$ 3,927.55$ & $\$ 9,826.02$ & $\$ 19,414.46$ & $\$ 17,733.42$ \\
\hline Total & & $\$ 7,286.80$ & $\$ 45,143.18$ & $\$ 89,731.78$ & $\$ 82,628.35$ \\
\hline
\end{tabular}

CI indicates cochlear implant; excl., excluding.

Sources: MBS Online, Australian Medicare Scheme. Accessed July 12, 2014. IHPA, National Hospital Cost Data Collection Australian Public Hospitals Cost report 2011-2012, Round 16, Appendix B (actual) for AR-DRG version 6.0x. Accessed July 14, 2014.

\section{ANALYTICAL METHODS}

The Markov model assumes five health states as illustrated in Figure 1. It was assumed all individuals with bilateral SP SNHL start in the HA state, and either transition to the unilateral CI health state, the simultaneous bilateral CI health state, or stay in the HA state. Once in the unilateral CI health state, individuals remain in that state or transition to the sequential bilateral health state, or the death state. Once in the sequential bilateral health state, they remain in that state or transition to the death state. Individuals in the simultaneous bilateral CI health state remain in that health state until their death. Total costs for the combined CI health states were weighted according to the proportion of the treated populations in each mutually exclusive CI health state.

Patients in the implant groups proceeded to a state where the implant was in place and functional. Over time, there is a risk that the implant will fail. In this patient, there is a possibility that a repeat implantation was conducted or that no further implantation took place (21).

The model followed a Markov process, allocating costs and utility to time spent in each cycle of 1 year. No reported deaths were caused by CI surgical procedures or any associated activities related to CI (21).

\section{Transition Probabilities}

The transition matrix depicting allowable transitions and probabilities are shown in Table 2. Transition probabilities were calculated from the percentage of adults treated in each CI treatment pathway. The following formula was used to calculate transition probabilities; $P=1-e^{-r t}$ where $P$ is the probability, $e$ is the base of the natural $\operatorname{logarithm}, r$ is the rate, and $t$ is the time period, which in this model is consistent with the cycle time of 1 year (15). The percentage of adults treated with unilateral $\mathrm{CI}$ in 2013 was $83.4 \%$ (10). The percentage treated with sequential bilateral $\mathrm{CI}$ was $16.3 \%$ and the percentage treated with simultaneous bilateral CI was $0.29 \%$ (10). Transition probabilities were assumed to remain constant, even though the percentage of adults treated bilaterally may increase in the future.

\section{Health Utilities for the Health States}

A QALY places a weight on time in different health states $(15,23)$. The health utilities that informed the base case model were taken from Chen et al. (11). Utility scores for simultaneous bilateral CI were not available for adults from Chen et al., so the same utility gains reported for sequential bilateral CI were applied to simultaneous bilateral CI. This was a gain in utility of 
TABLE 2. Transition matrix depicting allowable transitions and probabilities

\begin{tabular}{|c|c|c|c|c|c|c|}
\hline & & \multicolumn{5}{|c|}{ To $t+1$} \\
\hline & & Hearing Aid & Unilateral & Sequential & Simultaneous & Death \\
\hline \multirow[t]{5}{*}{ From $t$} & Hearing Aid & $1-(0.57+0.00287+0.04420)$ & 0.57 & 0 & 0.00287 & 0.04420 \\
\hline & Unilateral & 0 & $1-(0.15+0.04420)$ & 0.15 & 0 & 0.04420 \\
\hline & Sequential & 0 & 0 & $1-0.04420$ & 0 & 0.04420 \\
\hline & Simultaneous & 0 & 0 & 0 & $1-0.04420$ & 0.04420 \\
\hline & Death & 0 & 0 & 0 & 0 & 1 \\
\hline
\end{tabular}

$t$ indicates time.

0.035 from the unilateral CI state. The utility score for the HA state and the unilateral CI state was 0.495 and 0.765 , respectively (11). The World Health Organization recognizes Australia and Canada as high-income countries with similar health care systems and similar population demographics from a hearing loss perspective (24).

\section{Model Parameters}

Each time an adult transitioned from the HA state to a CI state, or from the unilateral CI state to the sequential bilateral CI state the presurgical assessment costs and surgical costs were counted. Maintenance costs for postsurgical assessment were assigned to each health state. They were assumed to exist annually for the rest of the adult's life. The cost of the replacement sound processor was averaged over 5 years to obtain an annual cost.

\section{Sensitivity Analyses}

To test for uncertainties in the model, changes were made to the following parameters: 1) the number of visits attended for pre and post assessment, 2) discount rates, 3) utility gains, 4) transition probabilities were varied to mirror the paediatric treatment mix which includes more surgeries for sequential bilateral and simultaneous bilateral CI, and 5) time horizons of 20 and 30 years.

\section{RESULTS}

\section{Incremental Costs per QALY}

The lifetime costs for bilateral HAs were AUD 14,254. The lifetime costs for the combined CI health states including weighted costs for unilateral, sequential bilateral, and simultaneous bilateral CI were AUD 34,541. The estimated cost/QALY for bilateral HAs was AUD
3,586/QALY. The ICUR for unilateral CI compared with bilateral HAs was AUD 9,799/QALY and simultaneous bilateral CI compared with bilateral HAs was AUD 26,765/QALY. When CI was compared at the decision point to treat surgically, the ICUR for CI compared with bilateral HAs was AUD 11,160/QALY. This includes the weighted mean costs of adults treated with unilateral CI, sequential bilateral $\mathrm{CI}$, and simultaneous bilateral $\mathrm{CI}$. These ICURS are reported in Table 3.

\section{Parameter Analyses-One-way Sensitivity}

Table 4 describes the impact on the model when certain parameters were varied. Changes occurred when the utility gains for bilateral CI were adjusted from 0.035 to $0.063(11,25)$. The ICUR decreased from AUD 11,160/ QALY to AUD 11,032/QALY for the combined CI health states compared with bilateral HAs.

When the assumed maximum visit frequency was applied to all patient visits, excluding HA patients whose visits were assumed to remain constant, the cost/QALY for unilateral CI increased to AUD 10,401/QALY when compared with bilateral HAs. The ICUR for the combined CI health states increased to AUD 11,813/QALY when compared with bilateral HAs. To model the possibility that adult treatment configurations could reach the same potential as Australian paediatrics, the transition probabilities were adjusted to $67 \%$ implanted unilaterally, with $33 \%$ progressing to sequential bilateral CI, and $33 \%$ implanted with simultaneous bilateral CI. The ICUR for unilateral CI compared with Bilateral HAs decreased to AUD 9,856/QALY, and the combined CI health states ICUR increased to AUD 15,054/QALY.

This model was stable for changes to utility gains. The model was driven by changes to assessment visits and changes to transition probabilities. This was expected, as

TABLE 3. Discounted costs per quality adjusted life year

\begin{tabular}{lcccr}
\hline Treatment Group & Utility & Costs & Incremental Utility Gains & Incremental Cost \\
\hline Hearing aid & 3.98 & 14,254 & - & - \\
Unilat CI vs HAs & 5.71 & 31,299 & 1.74 & 3,586 \\
SimBi CI vs HAs & 4.08 & 16,994 & 0.10 & 9,799 \\
CI cohort vs HAs & 5.79 & 34,541 & 1.82 & 2,740 \\
\hline
\end{tabular}

Costs and utilities were discounted at $5 \%$.

HAs indicates hearing aids; QALY, quality adjusted life years; SimBi CI, simultaneous bilateral cochlear implant; Unilat CI, unilateral cochlear implant; vs, versus. 
TABLE 4. Scenario and parameter analysis

\begin{tabular}{|c|c|c|c|c|c|c|}
\hline \multirow[b]{3}{*}{ Parameter Change } & \multicolumn{6}{|c|}{ Costs/QALY (\$) } \\
\hline & \multicolumn{2}{|c|}{ HAs } & \multicolumn{2}{|c|}{ Unilateral CI vs HAs } & \multicolumn{2}{|c|}{$\begin{array}{l}\text { Weighted Mean } \\
\text { Costs CI Cohort }\end{array}$} \\
\hline & Defined & PSA & Defined & PSA & Defined & PSA \\
\hline Basecase $^{a}$ & 3,586 & 3,608 & 9,799 & 9,610 & 11,160 & 10,846 \\
\hline Bilateral utility gain of 0.06 & 3,586 & 3,593 & 9,799 & 9,786 & 11,032 & 10,988 \\
\hline One visit per therapy & 3,227 & 3,198 & 9,525 & 9,499 & 10,781 & 10,817 \\
\hline Maximum visits ${ }^{\mathrm{b}}$ & 3,586 & 3,572 & 10,401 & 10,178 & 11,813 & 11,589 \\
\hline Discount $3 \%$ & 3,591 & 3,569 & 7,538 & 7,508 & 9,421 & 9,378 \\
\hline Discount $3.5 \%$ & 3,590 & 3,555 & 8,095 & 8,203 & 9,844 & 9,973 \\
\hline Discount $6 \%$ & 3,582 & 3,613 & 10,945 & 10,721 & 12,057 & 11,780 \\
\hline TPs equivalent to children ${ }^{c}$ & 3,586 & 3,567 & 9,856 & 9,983 & 15,054 & 15,195 \\
\hline 20-year time horizon & 3,576 & 3,548 & 14,268 & 14,511 & 16,166 & 16,363 \\
\hline 30-year time horizon & 3,581 & 3,578 & 11,941 & 12,061 & 13,644 & 13,757 \\
\hline
\end{tabular}

${ }^{\mathrm{a}}$ Base case assumed 8 pre- and 12 postassessment visits for CI in the first year. For HA, base case assumed four pre- and three postassessment visits in the first year.

${ }^{\mathrm{b}}$ Max assessment visits assumed 14 pre- and 18 postassessment visits for $\mathrm{CI}$ in the first year, HA visits were not changed in the sensitivity assessment of maximum visits.

${ }^{\mathrm{c}}$ In Australia $67 \%$ of children were implanted unilaterally, 33\% progressed to sequential bilateral CI, and 33\% were implanted with simultaneous bilateral CI.

HAs indicates hearing aids; PSA, probability sensitivity analysis; QALY, quality adjusted life years; TPs, transition probabilities.

more assessments would typically increase costs, and more adults treated with sequential bilateral and simultaneous bilateral CI would also typically increase costs.

The average age of adult and senior recipients in Australia was estimated to be approximately age 55 to 59 years (10). When the time horizon was shortened to 20 years, the ICUR for simultaneous bilateral CI increased to AUD 42,719/QALY compared with bilateral HAs, rendering the procedure marginally cost-effective. When placed in the context with other treatment options, the ICUR for the combined CI health states when compared with bilateral HAs was AUD 16,166/QALY.

When the time horizon was adjusted to 30 years, the ICUR for simultaneous bilateral CI when compared with bilateral HAs increased to AUD 34,483/QALY. For the combined CI health states the ICUR was AUD 13,644/ QALY compared with bilateral HAs.

\section{Sensitivity Analysis}

Probability sensitivity analysis was performed via 1,000 Monte Carlo simulations per health state. The results are reported in Table 4. All simulations were consistent with the determined outputs, such as the base case, reporting AUD 3,608/QALY for bilateral HAs. The ICUR for unilateral CI was AUD 9,610/QALY, and for the combined CI health states it was AUD 10,846/ QALY, respectively, when compared with bilateral HAs. This relative agreement between the determined and simulated outcomes demonstrates the model was robust.

The cost-effectiveness acceptability curves for simultaneous bilateral $\mathrm{CI}$, and unilateral $\mathrm{CI}$, compared with bilateral HAs appear in Figure 2. When a cost-effectiveness threshold of AUD 50,000/QALY was applied, it confirms the weighted CI treatment regime was cost-effective when compared with bilateral HAs.

\section{DISCUSSION}

CI treatments were considered in a continuum of care as surgical treatments consistent with eye laser surgery (26). This was appropriate because the treatment populations were mutually exclusive and the interventions were exhaustive (23). In a recent retrospective analysis of paediatric patients who qualified for bilateral CI treatment, only $52 \%$ received two implants (27) despite being referred for bilateral SP SNHL. Patients are heterogeneous in their clinical presentation. The proportion of patients, regardless of paediatric or adult, not treated bilaterally should be counted when assessing the cost-effectiveness of CI. The total mean costs should be weighted according to the proportion treated within each of the treatment groups. Previous studies assumed $100 \%$ of qualifying

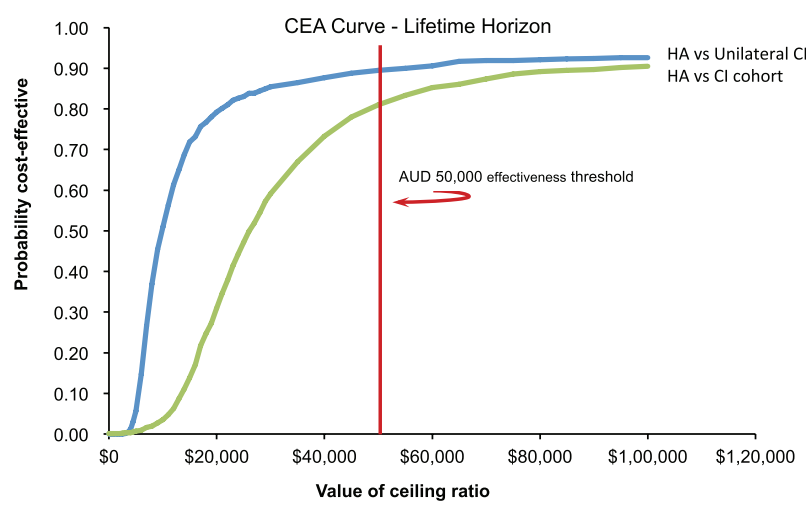

FIG. 2. Cost-effectiveness acceptability curves for simultaneous bilateral $\mathrm{Cl}$ and unilateral $\mathrm{Cl}$ compared with HAs with an AUD 50,000 effectiveness threshold. $\mathrm{Cl}$ indicates cochlear implantation; HAs, hearing aids. 
patients with bilateral SP SNHL would be treated with sequential or simultaneous bilateral CI $(5,11)$.

CI was cost effective as an alternative treatment option when compared with bilateral HAs, when a cost-effectiveness threshold of AUD 50,000/QALY was applied. There is a policy imperative to consider additional funding for sequential bilateral and simultaneous bilateral CI in the Australian public health care systems as adults with sequential bilateral and simultaneous bilateral CI have better outcomes than adults with unilateral $\mathrm{CI}$ or bilateral HAs $(7,9,14)$.

The number of pre and post-surgical visits attended by patients and the proportion of patients treated with sequential bilateral CI and simultaneous bilateral CI were the major drivers of this model. From Australian surgery data, it is highly unlikely that $100 \%$ of unilaterally treated adults will be treated with sequential bilateral CIs. This is a significant realization in understanding the economic costs of CI.

Failure rates and revision surgeries were assumed in all other economic evaluations. These consequences were available in Australia from published sources (21) and informed parameter inputs which improved the robustness and certainty of this model (21). They were not varied in the sensitivity analysis. This parameter input should be assessed for sensitivity when applying the model in another country. Nonimplant use remained steady in the model, as the rate was relatively low, and was not varied in the sensitivity analysis.

A lack of higher levels of evidence prevented an analysis from a societal perspective incorporating taxation transfers, productivity improvements, and reduced cost of illness. Fiscal models that examine potential increases in productivity arising from better communication and hearing abilities because of $\mathrm{CI}$ are needed.

Strategies that reduce the number of clinic visits are warranted. New sound processor technology and programming software that enables quicker fitting times would introduce cost efficiencies into clinical practice and reduce the overall costs associated with CI treatments. Future research that evaluates the potential cost savings of such technologies merits attention.

This evaluation is limited by the use of secondary sources for the utility gains and other parameter estimates. In the absence of any utility data from Australia, utilities were obtained from a Canadian study (11). Australia and Canada both share high income status from a World Health Organization perspective, suggesting that Canadian utilities were applicable to an Australian population (24). The gain for sequential bilateral CI was applied to the simultaneous bilateral procedure in the model because no published utilities were available for adult simultaneous bilateral CI. The utility gain from simultaneous bilateral CI was likely to be diluted as simultaneous implantation allowed for better surgical control compared with sequential implantation (28). Clinical studies with economic endpoints are required for a deeper understanding of the treatment benefits in the
Australian context. An important population subgroup are adults who use bimodal listening. Data on the percentage of Australian adults in this group were not available.

Health resource utilization was limited to CI procedures in the model because data was not available for wider resource utilization. Linked data to major claims databases, hospital records, disease registries, and primary care should be investigated to obtain a more accurate impact of hearing loss and $\mathrm{CI}$ on the health care system, and more broadly on society.

Acknowledgments: The authors sincerely acknowledge the contributions of Leslie Sosa-Ritchie, Dr. Yuan Cheng, Dr. Bayzid Rehman, Thathya V. Ariyaratne, Shaun Hand, Craig Stewart, and Prof. Quentin Summerfield for constructive criticisms that led to the formulation of this model's perspective.

\section{REFERENCES}

1. Davis A. Hearing in Adults: the Prevalence and Distribution of Hearing Impairment and Reported Hearing Disability in the MRC Institute of Hearing Research's National Study of Hearing. London, U.K.: Whurr Publishers; 1995.

2. Offeciers E, Morera C, Müller J, Huarte A, Shallop J, Cavalle L. International consensus on bilateral cochlear implants and bimodal stimulation. Acta Otolaryngol 2005;125:918.

3. Dunn CC, Tyler RS, Oakley S, Gantz BJ, Noble W. Comparison of speech recognition and localization performance in bilateral and unilateral cochlear implant users matched on duration of deafness and age at implantation. Ear Hear 2008;29:352.

4. van Schoonhoven J, Sparreboom M, van Zanten BG, et al. The effectiveness of bilateral cochlear implants for severe-to-profound deafness in adults: A systematic review. Otol Neurotol 2013;34: $190-8$.

5. Bond M, Mealing S, Anderson R, et al. The effectiveness and costeffectiveness of cochlear implants for severe to profound deafness in children and adults: A systematic review and economic model. Health Technol Assess 2009;13:1-330.

6. Summerfield A, Marshall DH, Barton GR, Bloor KE. A cost-utility scenario analysis of bilateral cochlear implantation. Arch Otolaryngol Head Neck Surg 2002;128:1255-62.

7. Lammers MJ, Heijden GJ, Pourier VE, Grolman W. Bilateral cochlear implantation in children: A systematic review and best evidence synthesis. Laryngoscope 2014;124:1694-9.

8. TGA. Australian Register of Therapeutic Goods. Australian Government Department of Health. July 18, 2014.

9. Birman CS, Gibson WP, Elliott EJ. Pediatric cochlear implantation: Associated with minimal postoperative pain and dizziness. Otol Neurotol 2015;36:220-2.

10. AIHW. Procedure data cubes. Australian Institute of Health and Welfare. August 21, 2015.

11. Chen JM, Amoodi H, Mittmann N. Cost utility analysis of bilateral cochlear implantation in adults: A health economic assessment from the perspective of a publicly funded program. Laryngoscope 2014;124:1452-8.

12. National Institute for Health and Care Excellence. Cochlear Implants for children and adults with severe to profound deafness. NICE Technology Appraisal Guidance. 2006. p. 166.

13. Hayes Inc. Cochlear Implants: Bilateral Versus Unilateral-A Health Technology Assessment Prepared for Washington State Health Care Authority. April 17, 2013.

14. Washington State Health Care Authority: Health Technology Assessment. Health Technology Clinical Committee Final Findings and Decision. 20130517A-Cochlear Implants: Bilateral versus Unilateral. 2013. 
15. Gray AM, Clarke PM, Wolstenholme JL, Wordsworth S. Applied Methods of Cost-Effectiveness Analysis in Healthcare. Oxford: Oxford University Press; 2010.

16. Quentin Summerfield A, Barton GR, Toner J, et al. Self-reported benefits from successive bilateral cochlear implantation in postlingually deafened adults: Randomised controlled trial. Int $J$ Audiol 2006;45 (suppl 1):99-107.

17. Husereau D, Drummond M, Petrou S, et al. Consolidated health economic evaluation reporting standards (CHEERS) statement. BMC Med 2013;11:80.

18. MBS. Medicare Benefits Schedule. August 21, 2014.

19. IHPA. National Hospital Cost Data Collection Australian Public Hospitals Cost report 2011-2012, Round 16, Appendix B (actual) for AR-DRG version 6.0x. 2014. IHPA.

20. Raine CH, Summerfield Q, Strachan DR, Martin JM, Totten C. The cost and analysis of nonuse of cochlear implants. Otol Neurotol 2008;29:221-4.

21. Wang JT, Wang AY, Psarros C, Cruz M. Rates of revision and device failure in cochlear implant surgery: A 30 year experience. Laryngoscope 2014;124:2393-9.
22. Australian Hearing. Australian Hearing. August 21, 2014.

23. Drummond MF. Methods for the Economic Evaluation of Health Care Programmes. Oxford: Oxford University Press; 2005.

24. Stevens G, Flaxman S, Brunskill E, Mascarenhas M, Mathers CD, Finucane M. Global and regional hearing impairment prevalence: an analysis of 42 studies in 29 countries. Eur J Public Health 2013;23:146-52.

25. Summerfield AQ, Lovett RES, Bellenger H, Batten G. Estimates of the cost-effectiveness of pediatric bilateral cochlear implantation. Ear Hear 2010;31:611-24.

26. Brown GC, Brown MM, Sharma S, Brown H, Tasman W. Incremental cost effectiveness of laser photocoagulation for subfoveal choroidal neovascularization. Ophthalmology 2000;107:1374-80.

27. Hanvey K. Paediatric unilateral implantation in an era of routine simultaneous bilateral implantation. Cochlear Implants Int 2015;16: S23-5.

28. Litovsky RY, Johnstone PM, Godar SP. Benefits of bilateral cochlear implants and/or hearing aids in children: Beneficios de los implantes cocleares bilaterales y/o auxiliares auditivos en nios. Int J Audiol 2006;45 (suppl 1):78-91. 